Rapid Reviews COVID-19

\title{
Reviews of "Exploratory analysis of immunization records highlights \\ decreased SARS-CoV-2 rates in individuals with recent non-COVID-19 vaccinations"
}

Andrew Wiese ${ }^{1}$, Shaun Truelove ${ }^{2}$

${ }^{1}$ Assistant Professor, VUMC, Health Policy, United States,

2Johns Hopkins University Bloomberg School of Public Health, International Health

Published on: Sep 22, 2020

DOI: $10.1162 / 2 \mathrm{e} 3983 f 5 . e b 13 \mathrm{~d} 03 \mathrm{f}$

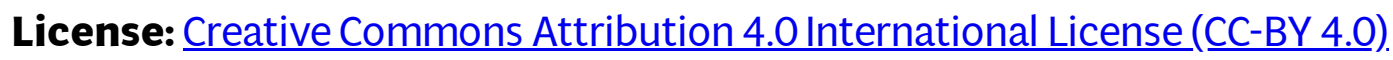


To read the original manuscript, click the link above.

Summary of Reviews: While the findings from this study are intriguing, the potential for spurious association between vaccination and infection is substantial. There are limitations to the data and findings could be misleading.

Reviewer 1 (Andrew Wiese)

Reviewer 2 (Shaun Truelove) | $\square \square \square \square \square$

\section{RR:C19 Strength of Evidence Scale Key}

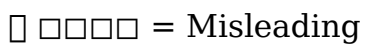

प्र $\square \square \square=$ Not Informative

प्रा पि = Potentially Informative

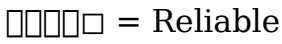

प्राप्र = Strong

To read the reviews, click the links below. 